\title{
CATEGORICAL REALIZATIONS OF QUIVERS
}

\author{
URIYA A. FIRST*
}

\begin{abstract}
We introduce and study categorical realizations of quivers. This construction generalizes comma categories and includes representations of quivers on categories, twisted representations of quivers (in the sense of 9]) and bilinear pairings as special cases. We prove a Krull-Schmidt Theorem in this general context, which results in a Krull-Schmidt Theorem for the special cases just mentioned. We also show that cancelation holds under milder assumptions. Using similar ideas we prove a version of Fitting's Lemma for natural transformations between functors.
\end{abstract}

\section{Overview}

Throughout, all rings are assumed to have a unity and ring homomorphisms are required to preserve it. Subrings are assumed to have the same unity as the ring containing them. The Jacobson radical of a ring $R$ is denoted by $\operatorname{Jac}(R)$.

In this paper, we introduce categorical realizations of quivers and representations of quivers on these realizations. This construction generalizes comma categories (which are a categorical realizations of the quiver $\bullet \rightarrow \bullet$; see [1, 3K]). Among its special cases are representations of quivers on additive categories (vector spaces in particular), twisted representations of quivers in the sense of 9, and bilinear pairings.

Using semi-centralizer subrings (also called semi-invariant subrings), introduced in [8, we establish a Krull-Schmidt Theorem for representations of quivers on their realizations, thus yielding Krull-Schmidt Theorems for the special cases mentioned above. We note that the categories in question are usually not abelian, so no "finite length" considerations (see Example 2.1) can be applied. Using similar ideas, we also prove a version of Fitting's Lemma for natural transformations between functors. Finally, different techniques are used to prove cancelation (from direct sums) of representations of quivers on their realizations under milder assumptions. Again, this yields cancelation theorems for the previous special cases.

This paper can be viewed as a continuation of 8 presenting further applications of semi-invariant subrings.

Section 2 recalls the Krull-Schmidt Theorem for pseudo-abelian categories. Section 3 introduces categorical realizations of quivers and section 4 defines linearly topologized categories. Section 5 recalls Fitting's Property (called quasi- $\pi_{\infty}$-regular

*Einstein Institute of Mathematics, Hebrew University of Jerusalem

E-mail address: uriya.first@gmail.com.

Date: August 6, 2018.

2010 Mathematics Subject Classification. 16G20, 16L30, 18E05.

Key words and phrases. quiver, additive category, pseudo-abelian category, Krull-Schmidt Theorem, Fitting's Lemma, Fitting's Property, semi-invariant subring, semi-centralizer subring, semiperfect ring.

This research was supported by an ERC grant \#226135 and by the Lady Davis Fellowship Trust. 
in [8) and semi-centralizer subrings. The main result of the paper and its applications are presented in section [6. A version of Fitting's Lemma for natural transformations is given in section 7 . Finally, section 8 discusses cancelation from direct sums.

\section{The Krull-Schmidt TheOrem}

Let $\mathscr{C}$ be an additive category. We say that the idempotents of $\mathscr{C}$ split or that $\mathscr{C}$ is pseudo-abelian if for all $C \in \mathscr{C}$ and an idempotent $e \in \operatorname{End}_{\mathscr{C}}(C)$, there exists an object $S \in \mathscr{C}$ and morphisms $i: S \rightarrow C, p: C \rightarrow S$ such that $i \circ p=e$ and $p \circ i=\mathrm{id}_{S}$. In this case, $(S, i, p)$ is called a summand of $C$. Every additive category is a full subcategory of a pseudo-abelian category obtained by artificially adding the nonexisting summands. This category is called the Karubi envelope of $\mathscr{C}$; see [10, Th. 6.10] for details.

An object of $C \in \mathscr{C}$ is said to have a Krull-Schmidt decomposition if $C=C_{1} \oplus$ $\cdots \oplus C_{t}$ with $C_{1}, \ldots, C_{t}$ indecomposable, and for every other such decomposition $C=C_{1}^{\prime} \oplus \cdots \oplus C_{t^{\prime}}^{\prime}$, we have $t=t^{\prime}$ and $C_{i} \cong C_{i}^{\prime}$ after suitable reordering of the $C_{i}$-s. We say that a pseudo-abelian category $\mathscr{C}$ is a Krull-Schmidt category (abbrev.: KS category) if every object in $\mathscr{C}$ has a Krull-Schmidt decomposition.

Recall that a ring $R$ is semiperfect if $R / \operatorname{Jac}(R)$ is semilocal and $\operatorname{Jac}(R)$ is idempotent lifting (e.g. if $\operatorname{Jac}(R)$ is nil). Equivalently, a ring $R$ is semiperfect if there are pairwise orthogonal idempotents $e_{1}, \ldots, e_{t}$ such that $\sum_{i} e_{i}=1$ and $e_{i} R e_{i}$ is local for all $i$; see [11, §2.7]. For example, if $R$ is semilocal and complete in the $\operatorname{Jac}(R)$-adic topology, i.e. $R=\lim _{\longleftarrow}\left\{R / \operatorname{Jac}(R)^{n}\right\}_{n \in \mathbb{N}}$, then $R$ is semiperfect.

Let $\mathscr{C}$ be a pseudo-abelian category. The Krull-Schmidt Theorem (which is also due to Azumaya and Remak; see [11, Th. 2.9.17 ff.]) asserts that an object $C \in \mathscr{C}$ has a Krull-Schmidt Decomposition when $\operatorname{End}_{\mathscr{C}}(C)$ is semiperfect. In particular, if all objects in $\mathscr{C}$ have a semiperfect endomorphism ring, then $\mathscr{C}$ is a KS category.

Example 2.1. Abelian categories in which all objects have finite length are KS categories. This follows from the Hadara-Sai Lemma (e.g. see [11, Prp. 2.9.29]1) which implies that the endomorphism ring of every indecomposable object of finite length is semiprimary.

\section{Categorical Realizations of Quivers}

In this section, we introduce categorical realizations of quivers. Many known objects are special cases of this construction: representations of quivers on categories, twisted representations of quivers in the sense of [9] and related papers, the comma category (e.g. see [1, §3K]), and even systems of bilinear pairings.

Recall that a quiver $Q$ is a directed graph (possibly with loops and multiple edges). We let $Q_{0}$ (resp. $Q_{1}$ ) denote the set of vertices (resp. directed edges) in $Q$. We always assume $Q_{0}$ is finite, but do not require $Q_{1}$ to be finite. The source (resp. target) of an edge $a \in Q_{1}$ is denoted by $s(a)$ (resp. $t(a)$ ). A path $p$ in $Q$ consists of a sequence $\left(v_{1}, a_{1}, v_{2}, a_{2}, \ldots, a_{n-1}, v_{n}\right)(n \geq 1)$ where $v_{1}, \ldots, v_{n} \in Q_{0}$ and $a_{i}$ is an edge from $v_{i}$ to $v_{i+1}$. In this case, we let $s(p)=v_{1}$ and $t(p)=v_{n}$. The concatenation of two paths is defined in the obvious way.

Fix a quiver $Q$ and assume that for every vertex $v \in Q_{0}$, we are given an additive category $\mathscr{C}_{v}$, and for every arrow $a \in Q_{1}$, we are given an additive category $\mathscr{C}_{a}$ and two additive functors $S_{a}: \mathscr{C}_{s(a)} \rightarrow \mathscr{C}_{a}, T_{a}: \mathscr{C}_{t(a)} \rightarrow \mathscr{C}_{a}$. The pair

$$
\mathscr{R}:=\left(\left\{\mathscr{C}_{x}\right\}_{x \in Q_{0} \cup Q_{1}},\left\{S_{a}, T_{a}\right\}_{a \in Q_{1}}\right)
$$

is called a categorical realization of $Q$.

\footnotetext{
1 The proof is phrased for modules but works in arbitrary abelian categories.
} 
A representation of $Q$ on $\mathscr{R}$ consists of a collection of objects $\left\{C_{v}\right\}_{v \in Q_{0}}$ and morphisms $\left\{f_{a}\right\}_{a \in Q_{1}}$ such that $C_{v} \in \mathscr{C}_{v}$ and $f_{a} \in \operatorname{Hom}_{\mathscr{C}_{a}}\left(S_{a} C_{s(a)}, T_{a} C_{t(a)}\right)$ for all $v \in Q_{0}$ and $a \in Q_{1}$. Denote by $\operatorname{Rep}(Q, \mathscr{R})$ the category of representations of $Q$ on $\mathscr{R}$. A morphism from $\left(\left\{C_{v}\right\},\left\{f_{a}\right\}\right)$ to $\left(\left\{C_{v}^{\prime}\right\},\left\{f_{a}^{\prime}\right\}\right)$ is a family of morphisms $\left\{\psi_{v}\right\}_{v \in Q_{0}}$ such that $\psi_{v} \in \operatorname{Hom}_{\mathscr{C}_{v}}\left(C_{v}, C_{v}^{\prime}\right)$, and for all $a \in Q_{1}$, the following diagram commutes:

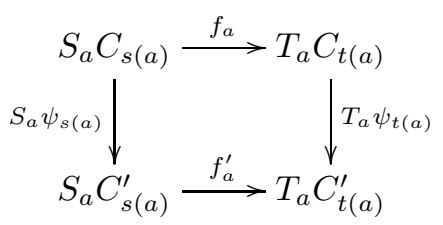

Example 3.1. When the categories $\left\{\mathscr{C}_{x}\right\}_{x \in Q_{0} \cup Q_{1}}$ are equal to a fixed additive category $\mathscr{C}$ and $S_{a}=T_{a}=\operatorname{id}_{\mathscr{C}}$ for all $a \in Q_{1}$, we get the notion of a representation of $Q$ on $\mathscr{C}$. The realization $\mathscr{R}$ is then called the trivial realization of $Q$ on $\mathscr{C}$, and we write $\operatorname{Rep}(Q, \mathscr{C})$ instead of $\operatorname{Rep}(Q, \mathscr{R})$. When $\mathscr{C}$ is the category of finite dimensional vector spaces over a field $F$, the representations of $Q$ on $\mathscr{C}$ are just representations of $Q$ over the field $F$ in the classical sense (see [2] for an extensive discussion about this case).

If $Q_{0}$ consists of a single vertex and $Q_{1}$ consists of $n$ arrows, then the isomorphism classes of $\operatorname{Rep}(Q, \mathscr{C})$ correspond to tuples of endomorphisms $\left(f_{1}, \ldots, f_{n}\right) \in$ End $_{\mathscr{C}}(C)$, considered up to simultaneous conjugacy. In fact, for $n=1, \operatorname{Rep}(Q, \mathscr{C})$ is equivalent to the category of endomorphisms in $\mathscr{C}$.

If $Q_{0}$ consists of two vertices $v_{1}, v_{2}$ and $Q_{1}$ consists of $n$ edges from $v_{1}$ to $v_{2}$, then the isomorphism classes of $\operatorname{Rep}(Q, \mathscr{C})$ correspond to tuples of homomorphisms $\left(f_{1}, \ldots, f_{n}\right) \in \operatorname{Hom}_{\mathscr{C}}\left(C, C^{\prime}\right)$ considered up to simultaneous composition on the left and on the right by an isomorphism in $\mathscr{C}$. In fact, when $n=1, \operatorname{Rep}(Q, \mathscr{C})$ is equivalent the category of morphisms in $\mathscr{C}$.

Example 3.2. Let $Q$ be the quiver $u \stackrel{a}{\rightarrow} v$ and let $\mathscr{R}$ be a categorical realization of $Q$. Then $\operatorname{Rep}(Q, \mathscr{R})$ is just the comma category $S_{a} \downarrow T_{a}$ (see [1, $\left.\S 3 \mathrm{~K}\right]$ ). The construction of $\operatorname{Rep}(Q, \mathscr{R})$ is therefore a generalization of comma categories.

Example 3.3. Let $R$ be a commutative ring and let $M:=\left\{M_{a}\right\}_{a \in Q_{1}}$ be a collection of right $R$-modules. Take $\mathscr{C}_{v}=\mathscr{C}_{a}=\operatorname{Mod}-R$ and define $S_{a}(X)=X \otimes_{R} M$, $T_{a}(X)=X$ for all $v \in Q_{0}, a \in Q_{1}, X \in \operatorname{Mod}-R$. Then $\operatorname{Rep}(Q, \mathscr{R})$ is the category of $M$-twisted representations of $Q$ on Mod- $R$ in the sense of [9, §2]. This example can be generalized by replacing $R$ with a ringed space $\left(X, \mathcal{O}_{X}\right)$ and Mod- $R$ with the category of sheaves of $\mathcal{O}_{X}$-modules.

Example 3.4. Let $R$ and $S$ be two rings and let $M$ be a fixed $(R, S)$-bimodule. An $(R, S)$-bilinear pairing (taking values in $M$ ) is a biadditive map $\omega: A \times B \rightarrow M$ with $A \in R$-Mod, $B \in \operatorname{Mod}-S$ satisfying $\omega(r a, b s)=r \cdot \omega(a, b) \cdot s$ for all $a \in A, b \in B$, $r \in R, s \in S$. Denote by $\mathscr{B}$ the class of $(R, S)$-bilinear pairings. We make $\mathscr{B}$ into a category by defining a homomorphism from $\omega: A \times B \rightarrow M$ to $\omega^{\prime}: A^{\prime} \times B^{\prime} \rightarrow M$ to be a pair $(f, g) \in \operatorname{Hom}_{R}\left(A, A^{\prime}\right) \times \operatorname{Hom}_{S}\left(B^{\prime}, B\right)$ such that

$$
\omega^{\prime}\left(f a, b^{\prime}\right)=\omega\left(a, g b^{\prime}\right) \quad \forall a \in A, b^{\prime} \in B^{\prime} .
$$

Composition is defined by the formula $\left(f^{\prime}, g^{\prime}\right) \circ(f, g)=\left(f^{\prime} \circ f, g^{\prime} \circ g\right)$. While the morphisms in $\mathscr{B}$ are not the standard morphisms of bilinear pairings, the isomorphism classes in $\mathscr{B}$ are still isomorphism classes of bilinear pairing in the usual sense.

We claim that the category $\mathscr{B}$ is equivalent $\operatorname{Rep}(Q, \mathscr{R})$ for suitable $Q$ and $\mathscr{R}$. Indeed, take $Q$ to be the quiver $u \stackrel{a}{\longrightarrow} v$, and define $\mathscr{R}$ by putting $\mathscr{C}_{u}=R$-Mod, $\mathscr{C}_{v}=(\operatorname{Mod}-S)^{\mathrm{op}}, \mathscr{C}_{a}=R$-Mod, $S_{a}(X)=X$ and $T_{a}(X)=\operatorname{Hom}_{S}(X, M)$. An object 
in $\operatorname{Rep}(Q, \mathscr{R})$ consists of a morphism of $R$-modules $A \rightarrow \operatorname{Hom}_{S}(B, M)$, whose datum is equivalent to an $(R, S)$-bilinear pairing $A \times B \rightarrow M$.

It is also possible to treat systems of $(R, S)$-bilinear pairings $\left\{\omega_{i}: A \times B \rightarrow\right.$ $\left.M_{i}\right\}_{i \in I}$ in this way (where $\left\{M_{i}\right\}_{i \in I}$ is a fixed family of $(R, S)$-bimodules). Replace $Q$ with a quiver consisting of two vertices $\{u, v\}$ and $|I|$ arrows $\left\{a_{i}\right\}_{i \in I}$ from $u$ to $v$, put $\mathscr{C}_{a_{i}}=R$-Mod and define $T_{a_{i}}(X)=\operatorname{Hom}_{S}\left(X, M_{i}\right)$. Alternatively, any system $\left\{\omega_{i}\right\}_{i \in I}$ is equivalent to an $(R, S)$-bilinear pairing taking values in $\prod_{i \in I} M_{i}$.

We record for later the following easy proposition.

Proposition 3.5. The category $\operatorname{Rep}(Q, \mathscr{R})$ is additive. If moreover $\mathscr{C}_{v}$ is pseudoabelian for all $v \in Q_{0}$, then $\operatorname{Rep}(Q, \mathscr{R})$ is pseudo-abelian.

Proof. The first assertion is straightforward. To see the second assertion, let $e:=\left\{e_{v}\right\}$ be an idempotent endomorphism of $\rho:=\left(\left\{C_{v}\right\},\left\{f_{a}\right\}\right) \in \operatorname{Rep}(Q, \mathscr{R})$. Then each $e_{v}$ is an idempotent endomorphism of $C_{v} \in \mathscr{C}_{v}$ and hence admits a corresponding summand $\left(X_{v}, i_{v}, p_{v}\right)$. Define $\sigma:=\left(\left\{X_{v}\right\},\left\{T_{a} p_{t(a)} \circ f_{a} \circ S_{a} i_{s(a)}\right\}_{a \in Q_{1}}\right)$, $i=\left\{i_{v}\right\}$ and $p=\left\{p_{v}\right\}$. We claim that $(\sigma, i, p)$ is a summand of $C$ corresponding to $e$. Namely, $i \in \operatorname{Hom}(\sigma, \rho), p \in \operatorname{Hom}(\rho, \sigma), i \circ p=e$ and $p \circ i=\operatorname{id}_{\sigma}=\left\{\operatorname{id}_{X_{v}}\right\}_{v \in Q_{0}}$. All these assertions follow by a routine argument. For example, $i \in \operatorname{Hom}(\sigma, \rho)$ since $T_{a} i_{t(a)} \circ\left(T_{a} p_{t(a)} \circ f_{a} \circ S_{a} i_{s(a)}\right)=T_{a}\left(i_{t(a)} \circ p_{t(a)}\right) \circ f_{a} \circ S_{a} i_{s(a)}=T_{a} e_{t(a)} \circ f_{a} \circ S_{a} i_{s(a)}=$ $f_{a} \circ S_{a} e_{s(a)} \circ S_{a} i_{s(a)}=f_{a} \circ S_{a}\left(e_{a} \circ i_{s(a)}\right)=f_{a} \circ S_{a} i_{s(a)}$.

Remark 3.6. If the categories $\left\{C_{x}\right\}_{x \in Q_{0} \cup Q_{1}}$ are abelian, the functors $\left\{S_{a}\right\}_{a \in Q_{1}}$ are right exact, and the functors $\left\{T_{a}\right\}_{a \in Q_{1}}$ are left exact, then $\operatorname{Rep}(Q, \mathscr{R})$ is abelian. This fails if we drop the exactness assumptions, though. We have omitted the straightforward technical verification of these facts since we do not need them here.

\section{Linearly Topologized Categories}

Recall that a topological ring $R$ is called linearly topologized (abbrev.: LT) if $R$ admits a local basis (i.e. a basis of neighborhoods of 0 ) consisting of two-sided ideals. For example, the $p$-adic integers $\mathbb{Z}_{p}$ are LT, but the $p$-adic numbers $\mathbb{Q}_{p}$ are not LT. In addition, every ring associated with the discrete topology is LT. When $R$ is LT, the topology on $R$ is completely determined by the set of open ideals in $R$, denoted $\mathcal{I}_{R}$. Conversely, every filter base ${ }^{2} \mathcal{F}$ of ideals of $R$ gives rise to a unique topology on $R$ such that $\mathcal{F}$ is a basis of neighborhoods of 0 ; take the topology spanned by $\{x+J \mid x \in R, J \in \mathcal{F}\}$. For an extensive discussion about topological rings and proofs of the previous facts, see [12].

Let $\mathscr{C}$ be an additive category. Recall that an ideal $I$ of $\mathscr{C}$ is a map adjoining to every two objects $X, Y \in \mathscr{C}$ an additive subgroup $I(X, Y) \subseteq \operatorname{Hom}_{\mathscr{C}}(X, Y)$ such that $\operatorname{Hom}_{\mathscr{C}}\left(Y, Y^{\prime}\right) \circ I(X, Y) \circ \operatorname{Hom}_{\mathscr{C}}\left(X^{\prime}, X\right) \subseteq I\left(X^{\prime}, Y^{\prime}\right)$ for all $X^{\prime}, Y^{\prime} \in \mathscr{C}$. For brevity, we write $I(X)$ instead of $I(X, X)$. The latter is clearly an ideal of $\operatorname{End}_{\mathscr{C}}(X)$.

A linearly topologized category consists of a pair $(\mathscr{C}, \mathcal{I})$ where $\mathscr{C}$ is an additive category and $\mathcal{I}$ is a collection of ideals of $\mathscr{C}$ such that for all $I, J \in \mathcal{I}$, there exists $K \in \mathcal{I}$ with $K(X, Y) \subseteq I(X, Y) \cap J(X, Y)$ for all $X, Y$. We then call $\mathcal{I}$ a linear topology on $\mathscr{C}$. In this case, for all $X, Y \in \mathscr{C}$, the cosets $\{f+I(X, Y) \mid f \in$ $\left.\operatorname{Hom}_{\mathscr{C}}(X, Y), I \in \mathcal{I}\right\}$ span a topology on $\operatorname{Hom}_{\mathscr{C}}(X, Y)$ which makes it into an additive topological group. Furthermore, composition is continuous, hence $\operatorname{End}_{\mathscr{C}}(X)$ is an LT ring for all $X \in \mathscr{C}$. We say that $(\mathscr{C}, \mathcal{I})$ is Hausdorff if $\bigcap_{I \in \mathcal{I}} I(X, Y)=0$ for all $X, Y \in \mathscr{C}$, i.e. the topology induced on $\operatorname{Hom}_{\mathscr{C}}(X, Y)$ is Hausdorff For all $X, Y \in \mathscr{C}$. If $\left(\mathscr{C}^{\prime}, \mathcal{I}^{\prime}\right)$ is another LT category, we say that an additive functor $F: \mathscr{C} \rightarrow \mathscr{C}^{\prime}$ is continuous if for all $X, Y \in \mathscr{C}$ and $I^{\prime} \in \mathcal{I}^{\prime}$, there exists $I \in \mathcal{I}$

\footnotetext{
${ }^{2}$ Let $X$ be a set. A nonempty subset $\mathcal{F} \subseteq P(X)$ is a filter base if $\emptyset \notin \mathcal{F}$ and for all $A, B \in \mathcal{F}$, there exists $C \in \mathcal{F}$ with $C \subseteq A \cap B$.
} 
such that $F(I(X, Y)) \subseteq I^{\prime}(F X, F Y)$. This is equivalent to saying that the map $F: \operatorname{Hom}_{\mathscr{C}}(X, Y) \rightarrow \operatorname{Hom}_{\mathscr{C}^{\prime}}(F X, F Y)$ is continuous for all $X, Y \in \mathscr{C}$.

We will often drop $\mathcal{I}$ from the notation, specifying only $\mathscr{C}$. In this case, we will write $\mathcal{I}_{\mathscr{C}}$ to denote the implicit collection $\mathcal{I}$.

Example 4.1. Any additive category $\mathscr{C}$ can be made into a Hausdorff LT category by taking $\mathcal{I}=\{0\}$ where $0(X, Y)=\left\{0_{X, Y}\right\}$ for all $X, Y \in \mathscr{C}$. In this case, any additive functor from $\mathscr{C}$ to another LT category is continuous.

Example 4.2. Let $R$ be an LT ring. Abusing the notation, for every $I \in \mathcal{I}_{R}$ and $M, N \in \operatorname{Mod}-R$, define $I(M, N)=\operatorname{Hom}_{R}(M, N I)$. Then $\left(\operatorname{Mod}-R, \mathcal{I}_{R}\right)$ is an LT category. We call $\mathcal{I}_{R}$ the standard linear topology of Mod- $R$.

We say that a module $M \in \operatorname{Mod}-R$ is Hausdorff if $\bigcap_{J \in \mathcal{I}_{R}} M J=0$. Let HMod- $R$ denote the full subcategory of Mod- $R$ whose objects are the Hausdorff modules. Then (HMod- $R, \mathcal{I}_{R}$ ) is a Hausdorff LT category. Note that HMod- $R$ contains the category of finitely generated projective right $R$-modules, denoted proj- $R$.

Let $\mathrm{fp}-R$ denote the category of finitely presented right $R$-modules and let hfp- $R$ denote the category of Hausdorff f.p. modules. Then $\left(\right.$ hfp- $\left.R, \mathcal{I}_{R}\right)$ is a pseudo-abelian Hasudorff LT category containing proj- $R$. The question of when $\mathrm{fp}-R=\mathrm{hfp}-R$ was considered in [8, §9]. Several cases when this holds are:

(i) $R$ is an almost fully-bounded noetherian ring whose primitive images are artinian (e.g. a noetherian PI ring) and $R$ is endowed with the $\operatorname{Jac}(R)$-adic topology ([11, Th. 3.2.28]).

(ii) $R$ is strictly pro-right artinian, namely, $R$ is the inverse limit of discrete right artinian rings $\left\{R_{i}\right\}_{i \in I}$ and the standard map $R \rightarrow R_{i}$ is surjective for all $i$ ([8, Cr. 9.8]). For example, this holds when $R$ is semilocal, $R=$ $\varliminf_{R}\left\{R / \operatorname{Jac}(R)^{n}\right\}_{n \in \mathbb{N}}$ and $\operatorname{Jac}(R)$ is f.g. as a right ideal.

(iii) $R$ is a complete rank-1 valuation ring ${ }^{3}$ considered as an LT using its valuation ([8, Rm. 9.7]).

Example 4.3. Let $R$ and $S$ be two LT rings and let $Q$ be an $(R, S)$-bimodule such that for all $J \in \mathcal{I}_{S}$ there is $I \in \mathcal{I}_{R}$ such that $I Q \subseteq Q J$. Then the functor $-\otimes_{R} Q:$ Mod- $R \rightarrow$ Mod- $S$ is continuous when both categories are endowed with the standard linear topology. Indeed, for $I$ and $J$ as above and $M, N \in$ Mod- $R$, we have $I(M, N) \otimes \operatorname{id}_{Q}=\operatorname{Hom}_{R}(M, N I) \otimes \operatorname{id}_{Q} \subseteq \operatorname{Hom}_{S}(M \otimes Q, N I \otimes Q) \subseteq$ $\operatorname{Hom}_{S}(M \otimes Q, N \otimes Q J)=J(M \otimes Q, N \otimes Q)$.

\section{Fitting's Property}

In this section, we recall the definition of Fitting's Property (abbrev.: FP) and bring several examples. Fitting's Property was introduced in [8, §5] under the name quasi- $\pi_{\infty}$-regular. The name was changed in recommendation of several experts due to the overusage of the term "regular" in ring theory.

Definition 5.1. A Hausdorff LT ring $R$ satisfies Fitting's Property (abbrev.: FP) if for all $n \in \mathbb{N}$ and $a \in \mathrm{M}_{n}(R)$, there is an idempotent $e \in \mathrm{M}_{n}(R)$ such that $a=e a e+(1-e) a(1-e)$, eae is invertible in $e R e$ and $(1-e) a^{n}(1-e) \rightarrow 0$ as $n \rightarrow \infty$ Here, $\mathrm{M}_{n}(R)$ is endowed with the product topology (view $\mathrm{M}_{n}(R)$ as $R^{n^{2}}$ ).

If the above condition is satisfied only when $n=1$, then $R$ is said to satisfy the weak Fitting Property.

The idempotent $e$ in the definition turns out to be uniquely determined by the element $a$ (see [8, Lm. 5.6]). We call $e$ the associated idempotent of a.

\footnotetext{
${ }^{3}$ To our purposes, a valuation ring $R$ is of rank 1 if its additive valuation takes values in $\mathbb{R}_{\geq 0}$.

4 This resembles Fitting's Lemma, hence the name "Fitting's Property".
} 
Example 5.2. (i) Let $R$ be a rank-1 valuation ring. Then $R$ is Henselian if and only if it has FP as an LT ring; see [8, Prp. 8.6]. Thus, Fitting's Property can be thought of as a generalization of Henselianity for topological rings.

(ii) A non-topological ring $R$ has FP when given the discrete topology if and only if it is (strongly) $\pi_{\infty}$-regular $\left[\right.$ ([8, Prp. 4.2]). The family of $\pi_{\infty}$-regular rings include semiprimary and right/left perfect ring 6 (6, Rm. 2.9]). In particular, discrete left/right artinian rings have FP.

(iii) An LT ring $R$ is called pro-semiprimary if it is the inverse limit of discrete semiprimary rings. For example, $\mathbb{Z}_{p}$ is pro-semiprimary since $\mathbb{Z}_{p} \cong \lim \left\{\mathbb{Z} / p^{n} \mathbb{Z}_{n \in \mathbb{N}}\right.$ (as topological rings). By [8, Lm. 5.14], every pro-semiprimary ring has FP.

(iv) A product of LT rings satisfying FP has FP when given the product topology.

Although not needed here, we note that an LT ring with FP is semiperfect if and only if $R$ does not contain an infinite set of pairwise orthogonal idempotents; see [8, Lm. 5.9].

Let $R$ be a Hausdorff LT ring and let $R_{0} \subseteq R$ be a subring. According to [8, §5], the subring $R_{0}$ is called a (topologically) semi-centralizer subring if there exists a Hausdorff LT ring $S$, containing $R$ as a topological ring, and a subset $X \subseteq S$ such that $R_{0}=\operatorname{Cent}_{R}(X):=\{r \in R: r x=x r \forall x \in X\}$. By [8, §5], we have:

Theorem 5.3. Let $R$ be LT ring satisfying FP. Then every semi-centralizer subring of $R$ has FP (w.r.t. the induced topology). If moreover $R$ is semiperfect, then every semi-centralizer subring $R_{0}$ of $R$ is semiperfect and satisfies $\operatorname{Jac}\left(R_{0}\right)^{n} \subseteq \operatorname{Jac}(R)$ for some $n$.

The condition $\operatorname{Jac}\left(R_{0}\right)^{n} \subseteq \operatorname{Jac}(R)$ in the theorem implies that the semi-centralizer subrings of semiprimary (resp. right perfect) rings are semiprimary (resp. right perfect).

We finish with recalling the following facts:

Proposition 5.4. Let $R$ and $S$ be Hausdorff LT rings, let $\varphi: R \rightarrow S$ be a continuous ring homomorphism, and let $S_{0} \subseteq S$ be a semi-centralizer subring of $S$. Then $\varphi^{-1}\left(S_{0}\right)$ is a semi-centralizer subring of $R$.

Proof. See [8, Cr. 3.2]. The proof is phrased for non-topological rings but works for topological rings by [8, Prp. 5.4].

Proposition 5.5. The intersection of semi-centralizer subrings is a semi-centralizer subring.

Proof. This follows from [8, Prp. 5.4(e)].

\section{Main Result}

Let $Q$ be a quiver and let $\mathscr{R}=\left(\left\{\mathscr{C}_{x}\right\}_{x \in Q_{0} \cup Q_{1}},\left\{S_{a}, T_{a}\right\}_{a \in Q_{1}}\right)$ be a categorial realization of $Q$. We say that $\mathscr{R}$ is pseudo-abelian if each of the categories $\left\{\mathscr{C}_{v}\right\}_{v \in Q_{0}}$ is psuedo-abelian, and say that $\mathscr{R}$ is linearly topologized if each of the categories $\left\{\mathscr{C}_{v}\right\}_{v \in Q_{0}}$ is linearly topologized. In the latter case, we make $\operatorname{Rep}(Q, \mathscr{R})$ into an LT category as follows: Write $\mathcal{I}_{v}=\mathcal{I}_{\mathscr{C}_{v}}$. For every $I:=\left\{I_{v}\right\}_{v \in Q_{0}}$ with $I_{v} \in \mathcal{I}_{v}$ and $\rho=\left(\left\{C_{v}\right\},\left\{f_{a}\right\}\right), \rho^{\prime}=\left(\left\{C_{v}^{\prime}\right\},\left\{f_{a}^{\prime}\right\}\right) \in \operatorname{Rep}(Q, \mathscr{R})$ define $I\left(\rho, \rho^{\prime}\right)=$ $\operatorname{Hom}\left(\rho, \rho^{\prime}\right) \cap \prod_{v \in Q_{0}} I_{v}\left(C_{v}, C_{v}^{\prime}\right)$. Then $\left\{\left\{I_{v}\right\}_{v \in Q_{0}} \mid I_{v} \in \mathcal{I}_{v}\right\}$ is a linear topology on $\operatorname{Rep}(Q, \mathscr{R})$. (In case $Q_{0}$ is not assumed to be finite, one should adjust the definition by assuming that $I_{v}=\operatorname{Hom}_{\mathscr{C}_{v}}$ for almost all $v \in Q_{0}$.) Notice that the

\footnotetext{
5 Another name in the literature is "completely $\pi$-regular".

${ }^{6}$ A ring is semiprimary (resp. right prefect) if it is semilocal and its Jacobson radical is nilpotent (resp. right T-nilpotent).
} 
topology on $\operatorname{Hom}\left(\rho, \rho^{\prime}\right)$ is the topology induced from the the product topology on $\prod_{v \in Q_{0}} \operatorname{Hom}_{\mathscr{C}_{v}}\left(C_{v}, C_{v}^{\prime}\right)$.

Assume $\mathscr{R}$ is LT. We say that $\mathscr{R}$ is Hausdorff if each of the LT categories $\left\{\mathscr{C}_{v}\right\}_{v \in Q_{0}}$ is Hausdorff, and for all $a \in Q_{1}, C \in \mathscr{C}_{s(a)}$ and $C^{\prime} \in \mathscr{C}_{t(a)}$ we have

$(*) 0=\bigcap_{\substack{I \in \mathcal{I}_{s(a)} \\ I^{\prime} \in \mathcal{I}_{t(a)}}}\left(T_{a} I^{\prime}\left(C^{\prime}\right) \circ \operatorname{Hom}_{\mathscr{C}_{a}}\left(S_{a} C, T_{a} C^{\prime}\right)+\operatorname{Hom}_{\mathscr{C}_{a}}\left(S_{a} C, T_{a} C^{\prime}\right) \circ S_{a} I(C)\right)$.

It is easy to see that in this case $\operatorname{Rep}(Q, \mathscr{R})$ is also Hausdorff (this is true even

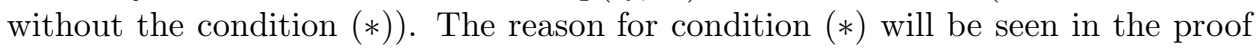
of Theorem 6.2 below.

Example 6.1. (i) If $\mathscr{R}$ is the trivial realization of $Q$ on a Hausdorff LT category $\mathscr{C}$, then $\mathscr{R}$ is Hausdorff (straightforward).

(ii) Assume that each of the categories $\left\{\mathscr{C}_{x}\right\}_{x \in Q_{0} \cup Q_{1}}$ is Hausdorff LT and that the functors $\left\{S_{a}, T_{a}\right\}_{a \in Q_{1}}$ are continuous. Then $\mathscr{R}$ is Hausdorff. Indeed, we only need to verify (図): Let $a \in Q_{1}$ and let $C \in \mathscr{C}_{s(a)}, C^{\prime} \in \mathscr{C}_{t(a)}$. Since $S_{a}$ and $T_{a}$ are continuous, for all $J \in \mathcal{I}_{a}$, there are $I \in \mathcal{I}_{s(a)}$ and $I^{\prime} \in \mathcal{I}_{t(a)}$ such that $S_{a} I(C) \subseteq J\left(S_{a} C\right)$ and $T_{a} I^{\prime}\left(C^{\prime}\right) \subseteq J\left(T_{a} C^{\prime}\right)$. Therefore,

$$
\begin{aligned}
T_{a} I^{\prime}\left(C^{\prime}\right) \circ \operatorname{Hom}_{\mathscr{C}_{a}}\left(S_{a} C, T_{a} C^{\prime}\right)+\operatorname{Hom}_{\mathscr{C}_{a}}\left(S_{a} C, T_{a} C^{\prime}\right) \circ S_{a} I(C) & \subseteq \\
J\left(T_{a} C^{\prime}\right) \circ \operatorname{Hom}_{\mathscr{C}_{a}}\left(S_{a} C, T_{a} C^{\prime}\right)+\operatorname{Hom}_{\mathscr{C}_{a}}\left(S_{a} C, T_{a} C^{\prime}\right) \circ J\left(S_{a} C\right) & \subseteq \\
J\left(S_{a} C, T_{a} C^{\prime}\right)+J\left(S_{a} C, T_{a} C^{\prime}\right) & =J\left(S_{a} C, T_{a} C^{\prime}\right) .
\end{aligned}
$$

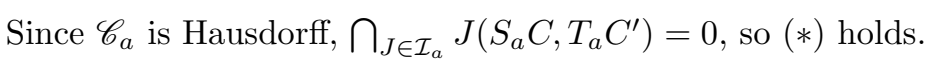

Theorem 6.2. Let $Q$ be a quiver and let $\mathscr{R}$ be a Hausdorff LT pseudo-abelian categorical realization such that $\operatorname{End}_{\mathscr{C}_{v}}(C)$ is semiperfect and has Fitting's Property for all $v \in Q_{0}, C \in \mathscr{C}_{v}$. Then $\operatorname{Rep}(Q, \mathscr{R})$ is a Hausdorff LT pseudo-abelian category and $\operatorname{End}(\rho)$ is semiperfect and has FP for all $\rho \in \operatorname{Rep}(Q, \mathscr{R})$. In particular, $\operatorname{Rep}(Q, \mathscr{R})$ is a Krull-Schmidt category.

Proof. That $\operatorname{Rep}(Q, \mathscr{R})$ is a Hausdorff LT category is clear. It is pseudo-abelian by Proposition 3.5. Thus, it is left to verify that for all $\rho=\left(\left\{C_{v}\right\},\left\{f_{a}\right\}\right) \in \operatorname{Rep}(Q, \mathscr{R})$, the endomorphism ring of $\rho$ is semiperfect and has FP. Recall that $\operatorname{End}(\rho)$ consists of collections $\left\{\psi_{v}\right\}_{v \in Q_{0}}$ such that $T_{a} \psi_{t(a)} \circ f_{a}=f_{a} \circ S_{a} \psi_{s(a)}$ for all $a \in Q_{1}$.

For every $a \in Q_{1}$, let $H_{a}=\operatorname{Hom}_{\mathscr{C}_{a}}\left(S_{a} C_{s(a)}, T_{a} C_{t(a)}\right)$. We make $H_{a}$ into a $\left(\operatorname{End}_{\mathscr{C}_{t(a)}}\left(C_{t(a)}\right), \operatorname{End}_{\mathscr{C}_{s(a)}}\left(C_{s(a)}\right)\right.$-bimodule by setting $f \cdot h \cdot g=T_{a} f \circ h \circ S_{a} g$ for all $f \in \operatorname{End}\left(C_{t(a)}\right), g \in \operatorname{End}\left(C_{s(a)}\right)$. For all $I \in \mathcal{I}_{s(a)}, J \in \mathcal{I}_{t(a)}$, let

$$
K_{I, J}=J\left(C_{t(a)}\right) \cdot H_{a}+H_{a} \cdot I\left(C_{s(a)}\right)
$$

and define

$$
\begin{aligned}
W_{a} & =\left[\begin{array}{cc}
\operatorname{End}\left(C_{t(a)}\right) & H_{a} \\
0 & \operatorname{End}\left(C_{s(a)}\right)
\end{array}\right] \quad \text { and } \\
\mathcal{B}_{a} & =\left\{\left[\begin{array}{cc}
J\left(C_{t(a)}\right) & K_{I, J} \\
0 & I\left(C_{s(a)}\right)
\end{array}\right] \mid I \in \mathcal{I}_{s(a)}, J \in \mathcal{I}_{t(a)}\right\} .
\end{aligned}
$$

Then $W_{a}$ is a ring and $\mathcal{B}_{a}$ is a filter base of ideals of $W_{a}$. Thus, $\mathcal{B}_{a}$ induces a linear ring topology on $W_{a}$, and that topology is Hausdorff since $\mathscr{R}$ is Hausdorff $\left(\bigcap_{I, J} K_{I, J}=0\right.$ by (因) $)$.

Let $R=\prod_{v \in Q_{0}} \operatorname{End}_{\mathscr{C}}\left(C_{v}\right)$. Then $R$ is a Hausdorff LT ring since $\mathscr{R}$ is Hausdorff. Define $\varphi_{a}: R \rightarrow W_{a}$ by

$$
\varphi_{a}\left(\left\{\psi_{v}\right\}\right)=\left[\begin{array}{cc}
\psi_{t(a)} & 0 \\
0 & \psi_{s(a)}
\end{array}\right]
$$


and let

$$
\alpha_{a}=\left[\begin{array}{cc}
0 & f_{a} \\
0 & 0
\end{array}\right] \in W_{a} .
$$

It is clear that $\varphi_{a}$ is a continuous homomorphism of LT rings. Furthermore, by definition, we have $\operatorname{End}(\rho)=\bigcap_{a \in Q_{1}} \varphi_{a}^{-1}\left(\operatorname{Cent}_{W_{a}}\left(\alpha_{a}\right)\right)$. Since $\operatorname{Cent}_{W_{a}}\left(\alpha_{a}\right)$ is a semi-centralizer subring of $W_{a}, \varphi_{a}^{-1}\left(\operatorname{Cent}_{W_{a}}\left(\alpha_{a}\right)\right)$ is a semi-centralizer subring of $R$ (Proposition 5.4), so by Proposition 5.5, $\operatorname{End}(\rho)$ is a semi-centralizer subring of $R$. The ring $R$ is semiperfect and has FP by assumption. Thus, by Theorem 5.3 , $\operatorname{End}(\rho)$ is semiperfect and has FP.

Remark 6.3. (i) If we drop the assumption that $Q_{0}$ is finite and that the endomorphism rings of objects in $\mathscr{C}_{v}\left(v \in Q_{0}\right)$ are semiperfect in Theorem 6.2, then the endomorphism rings of objects in $\operatorname{Rep}(Q, \mathscr{R})$ still have FP.

(ii) When the assumptions of Theorem 6.2 are satisfied, the indecomposable representations in $\operatorname{Rep}(Q, \mathscr{R})$ are precisely those with local endomorphism ring. (Indeed, an object in an additive category is indecomposable precisely when its endomorphism ring has no nontrivial idempotents, and a semiperfect ring with nontrivial idempotents is local.)

Example 6.4. Theorem 6.2 fails if we do not assume condition (函): Let $R$ and $S$ be two semiperfect LT rings satisfying FP, and assume that there is a non-topological ring $M$ containing $R$ and $S$ such that $R \cap S$ is not semiperfect. This implies that $R \cap S$ does not have FP with respect to any topology, for otherwise, $R \cap S$ would be semiperfect by [8, Lm. 5.9(ii)].

View $M$ as an $(R, S)$ bimodule and define $Q$ and $\mathscr{R}$ as in Example 3.4 with the difference that only projective $R$ - and $S$-modules are allowed. Namely, $Q$ is the quiver $u \stackrel{a}{\rightarrow} v, \mathscr{C}_{u}=R$-proj, $\mathscr{C}_{v}=(\operatorname{proj}-S)^{\mathrm{op}}, \mathscr{C}_{a}=R$-Mod, $S_{a}=$ id and $T_{a}=\operatorname{Hom}_{S}(-, M)$. Then $\mathscr{C}_{u}$ and $\mathscr{C}_{v}$ are Hausdorff with respect to their standard linear topology. Let $\rho=\left(\left\{C_{u}, C_{v}\right\},\left\{f_{a}\right\}\right) \in \operatorname{Rep}(Q, \mathscr{R})$ be the object corresponding to the $(R, S)$-bilinear pairing $\omega: R \times S \rightarrow M$ given by $\omega(r, s)=r s$. That is, $C_{u}={ }_{R} R, C_{v}=S_{S}$ and $f_{a}:{ }_{R} R \rightarrow \operatorname{Hom}_{S}\left(S_{S}, M_{S}\right) \cong M$ is just the inclusion map. In this case, $H_{a}=\operatorname{Hom}_{R}\left({ }_{R} R,{ }_{R} M\right) \cong M$ as an $(R, S)$-bimodule and under that isomorphism, $f_{a}$ is mapped to 1 . Following the proof of Theorem 6.2, we now see that $\operatorname{End}(\rho)$ is isomorphic to

$$
\left\{\left[\begin{array}{ll}
r & 0 \\
0 & s
\end{array}\right] \in\left[\begin{array}{ll}
R & M \\
0 & S
\end{array}\right] \mid\left[\begin{array}{ll}
r & 0 \\
0 & s
\end{array}\right]\left[\begin{array}{ll}
0 & 1 \\
0 & 0
\end{array}\right]=\left[\begin{array}{ll}
0 & 1 \\
0 & 0
\end{array}\right]\left[\begin{array}{ll}
r & 0 \\
0 & s
\end{array}\right]\right\} \cong R \cap S
$$

which is not semiperfect nor has FP. An explicit example of $R, S$ and $M$ as above is $R=\mathbb{Z}_{p}, S=\mathbb{Z}_{q}$ and $M=\mathbb{Q}_{p} \otimes_{\mathbb{Q}} \mathbb{Q}_{q}$. Here, $p, q$ are distinct prime numbers and $\mathbb{Z}_{p}$ and $\mathbb{Z}_{q}$ are identified with $\mathbb{Z}_{p} \otimes 1$ and $1 \otimes \mathbb{Z}_{q}$. Since $a \otimes 1=1 \otimes b$ implies $a=b \in \mathbb{Q}$, we see that $R \cap S=\left\{n m^{-1} \mid n \in \mathbb{Z}, m \in \mathbb{Z} \backslash(p \mathbb{Z} \cup q \mathbb{Z})\right\}$, a non-semiperfect ring.

The rest of this section concerns applications of Theorem 6.2 Recall that for an LT ring $R, \mathrm{fp}-R$ denotes the category of finitely presented right $R$-modules and hfp- $R$ denotes the category of Hausdorff finitely presented right $R$-modules.

Example 6.5. Let $\mathscr{R}$ be the trivial realization of $Q$ over an additive category $\mathscr{C}$ (i.e. $\mathscr{C}_{x}=\mathscr{C}$ and $S_{a}=T_{a}=\mathrm{id}_{\mathscr{C}}$ for all $x \in Q_{0} \cup Q_{1}, a \in Q_{1}$ ). The assumptions of Theorem 6.2 are satisfied for $\mathscr{R}$ when $\mathscr{C}$ is a Hausdorff LT category whose endomorphisms are semiperfect and has FP. These conditions are satisfied by the following categories:

(i) The category hfp- $R$, where $R$ is a semiperfect Hausdorff LT ring satisfying Fitting's Property. Indeed, by [8, Th. 8.3(ii)], the endomorphism ring of every object in hfp- $R$ is semiperfect and has FP. Note that hfp- $R$ contains all f.g. projective right $R$-modules.

(ii) The category fp- $R$ where $R$ is a strictly pro-right-artinian ring or a rank-1 Henselian valuation ring. This follows from (i) and Example 4.2 . 
(iii) The category $\operatorname{Rep}\left(Q^{\prime}, \mathscr{R}^{\prime}\right)$ where $\mathscr{R}^{\prime}$ is a categorical realization of $Q^{\prime}$ satisfying the assumptions of Theorem 6.2

Example 6.6. Let $R$ be a commutative LT ring with FP and let $M=\left\{M_{a}\right\}_{a \in Q_{1}}$ be a family of modules in hfp- $R$. Then the Krull-Schmidt Theorem holds for the category of $M$-twisted representations of $Q$ on hfp- $R$ in the sense of $9, \S 2]$. Indeed, apply Theorem 6.2 with the categorical realization of Example 3.3. The assumptions of Theorem 6.2 apply since the functors $\left\{T_{a}, S_{a}\right\}$ are continuous (Example 4.3) and the categories $\left\{\mathscr{C}_{x}\right\}_{x \in Q_{0} \cup Q_{1}}$ are Hasudorff LT (Example 6.5(i)).

Example 6.7. Let $R$ be a semiperfect LT ring satisfying FP. Then every endomorphism $f$ in hfp- $R$ decomposes to a direct sum of indecomposable endomorphism $f=f_{1} \oplus \cdots \oplus f_{t}$ and this decomposition is essentially unique, i.e. every other such decomposition is conjugate to the original one. (This decomposition can be regarded as a "Jordan decomposition" of $f$.) To see why this holds, recall from Example 3.1 that the category of endomorphisms in hfp- $R$ can be understood as $\operatorname{Rep}(Q, \operatorname{hfp}-R)$ for suitable $Q$. Now apply Theorem 6.2 together with the Krull-Schmidt Theorem; the assumptions of Theorem 6.2 hold by Example 6.5(i).

Example 6.8. Let $\mathscr{R}$ be a non-linearly-topologized semi-abelian categorical realization of $Q$. Assume that $\operatorname{End}_{\mathscr{C}_{v}}(C)$ is semiprimary for all $v \in Q_{0}$ and $C \in \mathscr{C}_{v}$. Then the endomorphism rings of all objects in $\operatorname{Rep}(Q, \mathscr{R})$ are semiprimary. Indeed, give the categories $\left\{\mathscr{C}_{x}\right\}_{x \in Q_{0} \cup Q_{1}}$ the discrete topology of Example 4.1. By Example 6.1] $\mathscr{R}$ is Hausdorff, and by Example [5.2(ii), the endomorphism rings of objects in $\mathscr{C}_{v}$ are semiprefect and have FP. Thus, we can apply Theorem 6.2 to $\mathscr{R}$. In the proof of the theorem, it was shown that the endomorphism ring of $\rho=\left(\left\{C_{v}\right\},\left\{f_{a}\right\}\right) \in \operatorname{Rep}(Q, \mathscr{R})$ is a semi-centralizer subring of $\prod_{v} \operatorname{End}_{\mathscr{C}_{v}}\left(C_{v}\right)$, which is semiprimary in our case. Therefore, by the comment after Theorem 5.3 . $\operatorname{End}(\rho)$ is semiprimary. (The same argument works if we replace semiprimary with right perfect.)

Examples of categories $\mathscr{C}_{v}$ with semiprimary endomorphisms rings include abelian categories consisting of objects of finite length (Example 2.1) and the category fp- $R$ when $R$ is a semiprimary ring (this is due to Bjork [4, Th. 4.1]; see [8, Ths. $7.3 \&$ 8.3] for generalizations). When the the categories $\left\{\mathscr{C}_{x}\right\}_{x \in Q_{0} \cup Q_{1}}$ are abelian, consist of objects of finite length, and the functors $\left\{S_{a}\right\}$ and $\left\{T_{a}\right\}$ are right-exact and left-exact, respectively, the category $\operatorname{Rep}(Q, \mathscr{R})$ is abelian and consists of objects of finite length. Therefore, can use Example 2.1 rather than Theorem 6.2 to prove that endomorphism rings in $\operatorname{Rep}(Q, \mathscr{R})$ are semiprimary.

Corollary 6.9. Let $R, S$ be semiperfect $L T$ rings satisfying $F P$ and let $M$ be an $(R, S)$-bimodule such that $\bigcap_{I, J}(I M+M J)=0$ where $I$ and $J$ range over $\mathcal{I}_{R}$ and $\mathcal{I}_{S}$, respectively. Then every $(R, S)$-bilinear pairing $\omega: A \times B \rightarrow R$ with $A \in R$-hfp and $B \in \mathrm{hfp}-S$ can be written as an orthogonal sum of indecomposable bilinear parings $\omega=\omega_{1} \perp \cdots \perp \omega_{t}$ and this decomposition is essentially unique. Namely, if $\omega=\omega_{1}^{\prime} \perp \cdots \perp \omega_{t}^{\prime}$ with $\omega_{1}^{\prime}, \ldots, \omega_{t}^{\prime}$ indecomposable, then $t=t^{\prime}$, and after suitable reordering, $\omega_{i}$ is isometric to $\omega_{i}$ for all $1 \leq i \leq t \overline{7}$

Proof. Define $\mathscr{R}$ and $Q$ as in Example 3.4 but take $\mathscr{C}_{u}$ and $\mathscr{C}_{v}$ to be $R$-hfp and hfp- $S$ instead of $R$-Mod and Mod- $S$. Then the objects of $\operatorname{Rep}(Q, \mathscr{R})$ correspond to $(R, S)$-bilinear pairings $\omega: A \times B \rightarrow M$ with $A \in$ hfp- $R$ and $B \in$ hfp- $S$.

Recall that $Q$ is the quiver $u \stackrel{a}{\rightarrow} v$. The category $\mathscr{C}_{u}=$ hfp- $R$ is Hausdorff LT (when given the standard topology) by definition. Likewise, the category $\mathscr{C}_{v}=$ $(\text { hfp- } S)^{\text {op }}$ inherits the standard (Hausdorff) linear structure of hfp- $S$. We claim

\footnotetext{
7 Two $(R, S)$-bilinear pairings $\omega_{i}: A_{i} \times B_{i} \rightarrow M(i=1,2)$ are isometric if there are isomorphisms $f: A_{1} \rightarrow A_{2}$ and $g: B_{1} \rightarrow B_{2}$ such that $\omega_{2}(f a, g b)=\omega_{1}(a, b)$ for all $a \in A_{1}, b \in B_{1}$.
} 
that $\mathscr{R}$ is LT Hausdorff, namely, that (函) holds. Let $I \in \mathcal{I}_{R}$ and $J \in \mathcal{I}_{S}$. Then for all $A \in R$-Mod, $B \in \operatorname{Mod}-S$, we have

$$
\begin{aligned}
\operatorname{Hom}_{\mathscr{C}_{a}}\left(S_{a} A, T_{a} B\right) \circ S_{a} I(A) & =\operatorname{Hom}_{R}\left(A, \operatorname{Hom}_{S}(B, M)\right) \circ I(A) \\
& \subseteq I\left(A, \operatorname{Hom}_{S}(B, M)\right)=\operatorname{Hom}_{R}\left(A, I \cdot \operatorname{Hom}_{S}(B, M)\right) \\
& \subseteq \operatorname{Hom}_{R}\left(A, \operatorname{Hom}_{S}(B, I M)\right)
\end{aligned}
$$

and

$$
\begin{aligned}
T_{a} J(B) \circ \operatorname{Hom}_{\mathscr{C}_{a}}\left(S_{a} A, T_{a} B\right) & =\operatorname{Hom}_{S}(-, M)(J(B, B)) \circ \operatorname{Hom}_{R}\left(A, \operatorname{Hom}_{S}(B, M)\right) \\
& \subseteq \operatorname{Hom}_{R}\left(A, \operatorname{Hom}_{S}(B, M J)\right) .
\end{aligned}
$$

The last inclusion holds since for all $f \in J(B, B), g \in \operatorname{Hom}_{R}\left(A, \operatorname{Hom}_{S}(B, M)\right)$ and $a \in A$, we have $\left(\operatorname{Hom}_{S}(f, M) \circ g\right) a=(g a) \circ f \in J(B, M)=\operatorname{Hom}_{S}(B, M J)$. Вy assumption, $\bigcap_{I, J}(I M+M J)=0$, so the previous two equations imply (荬).

Next, observe that By Example 6.5(i), each of objects in $\mathscr{C}_{u}$ and $\mathscr{C}_{v}$ has a semiperfect endomorphism ring satisfying FP. Thus, by Theorem 6.2 , the objects of $\operatorname{Rep}(Q, \mathscr{R})$ have a Krull-Schmidt decomposition. We are therefore finished if we show that direct sum decompositions in $\operatorname{Rep}(Q, \mathscr{R})$ correspond to orthogonal sum decompositions of $(R, S)$-bilinear pairings. (This is not obvious since not all morphisms in $\operatorname{Rep}(Q, \mathscr{R})$ correspond to morphisms of $(R, S)$-bilinear pairings; see Example 3.4.) This is routine and is left to the reader.

\section{Quivers with Relations And Categories}

In this section we consider quivers with relations and thus restrict our discussion to trivial realizations of quivers on a fixed additive category $\mathscr{C}$.

For a quiver $Q$, denote by $P(Q)$ the set of paths in $Q$. For $\left(\left\{C_{v}\right\},\left\{f_{a}\right\}\right) \in$ $\operatorname{Rep}(Q, \mathscr{C})$ and a path $p=\left(v_{1}, a_{1}, v_{2}, a_{2}, \ldots, v_{n}\right)$, we set $f_{p}=f_{a_{n-1}} \circ \cdots \circ f_{a_{1}}$ if $n>1$ and $f_{p}=\operatorname{id}_{C_{v_{1}}}$ if $n=1$. A path related quiver is a quiver $Q$ equipped with a family of pairs $\mathfrak{R}=\left\{p_{i}, q_{i}\right\}_{i \in I}$ such that $p_{i}$ and $q_{i}$ are paths in $Q$ admitting a common source and target. A representation of $(Q, \mathfrak{R})$ on $\mathscr{C}$, is a representation $\left(\left\{C_{v}\right\},\left\{f_{a}\right\}\right) \in$ $\operatorname{Rep}(Q, \mathscr{C})$ such that $f_{p_{i}}=f_{q_{i}}$ for all $i \in I$. The class of representations of $(Q, \mathfrak{R})$ on $\mathscr{C}$ forms a full subcategory of $\operatorname{Rep}(Q, \mathscr{C})$ and thus, Theorem 6.2 remains true if we replace $\operatorname{Rep}(Q, \mathscr{C})$ with that category.

Every category $\mathscr{U}$ with finitely many objects can be considered as a path related quiver. Indeed, take $Q_{0}$ to be the objects of $\mathscr{U}$, let $Q_{1}$ be the morphisms of $\mathscr{U}$, and take $\mathfrak{R}$ to be the relations induced from the composition rule. In this case, a representation of $(Q, \mathfrak{R})$ on $\mathscr{C}$ is merely a functor from $\mathscr{U}$ to $\mathscr{C}$. Theorem 6.2 now reads as:

Theorem 7.1. Let $\mathscr{U}$ be a category with finitely many objects and let $\mathscr{C}$ be a Hausdorff LT pseudo-abelian category such that $\operatorname{End}_{\mathscr{C}}(C)$ is semiperfect and has $F P$ for all $C \in \mathscr{C}$. Then the category of functors $F: \mathscr{U} \rightarrow \mathscr{C}$, denoted $\operatorname{Hom}(\mathscr{U}, \mathscr{C})$, is a Hausdorff LT pseudo-abelian category and $\operatorname{End}(F)$ is semiperfect and has FP for all $F \in \operatorname{Hom}(\mathscr{U}, \mathscr{C}) \&$ In particular, $\operatorname{Hom}(\mathscr{U}, \mathscr{C})$ is a Krull-Schmidt category.

In case $\mathscr{U}$ is an infinite small category, the same argument shows that $\operatorname{End}(F)$ has FP for every functor $F: \mathscr{U} \rightarrow \mathscr{C}$. While this statement is meaningless in case $\mathscr{U}$ is a large category ( $\operatorname{since} \operatorname{End}(F)$ is not necessarily a set), we can still get a statement resembling Fitting's Lemma:

\footnotetext{
8 Recall that an endomorphism of a functor $F$ is just a natural transformation from $F$ to itself. Namely, it is a collection $\left\{t_{U}\right\}_{U \in \mathscr{U}}$ with $t_{U} \in \operatorname{End}_{\mathscr{C}}(F U)$ such that for every $U, V \in \mathscr{U}$ and $f \in \operatorname{Hom}_{\mathscr{U}}(U, V)$, one has $t_{V} \circ F f=F f \circ t_{U}$. When $\mathscr{U}$ is a small category (i.e. the objects of $\mathscr{U}$ form a set), $\operatorname{End}(F)$ is a set.
} 
Theorem 7.2. Let $\mathscr{U}$ be any category and let $\mathscr{C}$ be an additive Hausdorff LT pseudo-abelian category such that $\operatorname{End}_{\mathscr{C}}(C)$ has $F P$ for all $C \in \mathscr{C}$. Let $F: \mathscr{U} \rightarrow \mathscr{C}$ be a functor and let $t: F \rightarrow F$ be a natural transformation. Then there are functors $F_{0}, F_{1}: \mathscr{U} \rightarrow \mathscr{C}$ and natural transformations $t_{0}: F_{0} \rightarrow F_{0}, t_{1}: F_{1} \rightarrow F_{1}$ such that $F=F_{0} \oplus F_{1}$ (i.e. $F U=F_{0} U \oplus F_{1} U$ for all $\left.U \in \mathscr{U}\right), t=t_{0} \oplus t_{1}$ (i.e. $t_{U}=\left(t_{0}\right)_{U} \oplus\left(t_{1}\right)_{U}$ for all $\left.U \in \mathscr{U}\right), t_{1}$ is a natural isomorphism, and $\left(\left(t_{0}\right)_{U}\right)^{n} \rightarrow 0$ as $n \rightarrow \infty$ in $\operatorname{End}_{\mathscr{C}}\left(F_{0} U\right)$ for all $U \in \mathscr{U}$. The decomposition $F=F_{0} \oplus F_{1}$ is unique up to natural isomorphism 9

Proof. Let $\mathscr{U}^{\prime}$ be a finite full subcategory of $\mathscr{U}$, and let $F^{\prime}=\left.F\right|_{\mathscr{U}^{\prime}}$ and $t^{\prime}=\left.t\right|_{\mathscr{U}^{\prime}}$. By Theorem 7.1 and Remark 6.3(i), End $\left(F^{\prime}\right)$ is a has FP (when endowed with the topology induced from the product topology on $\prod_{U \in \mathscr{U}} \operatorname{End}_{\mathscr{C}}\left(F^{\prime} U\right)$ ). Therefore, $t^{\prime}$ admits an associated idempotent $e^{\prime} \in \operatorname{End}\left(F^{\prime}\right)$ (see section 5 ), namely, $t^{\prime}=$ $e^{\prime} t^{\prime} e^{\prime}+\left(1-e^{\prime}\right) t^{\prime}\left(1-e^{\prime}\right), e^{\prime} t^{\prime} e^{\prime}$ is invertible in $e^{\prime} \operatorname{End}\left(F^{\prime}\right) e^{\prime}$ and $\left(1-e^{\prime}\right) t^{\prime n}\left(1-e^{\prime}\right) \rightarrow 0$ when $n \rightarrow \infty$.

Let $\mathscr{U}^{\prime \prime}$ be another finite full subcategory of $\mathscr{U}$. Define $F^{\prime \prime}$ and $e^{\prime \prime}$ analogously to $F^{\prime}$ and $e^{\prime}$. If $\mathscr{U}^{\prime \prime}$ contains $\mathscr{U}^{\prime}$, then $\left.e^{\prime \prime}\right|_{\mathscr{U}^{\prime}}$ is also an associated idempotent for $t^{\prime}$, so the the uniqueness of the associated idempotent implies $\left.e^{\prime \prime}\right|_{\mathscr{U}}=e^{\prime}$. Using this, we may define a natural transformation $e: F \rightarrow F$ by letting $e_{U}$ to be $e_{U}^{\prime}$ where $e^{\prime}$ is obtained from some finite subcategory $\mathscr{U}^{\prime}$ as above that contains $U$. It is clear that $e$ is idempotent, $t=e t e+(1-e) t(1-e)$, and for all $U \in \mathscr{U}, e_{U} t_{U} e_{U}$ is invertible in $e_{U} \operatorname{End}_{\mathscr{C}}(F U) e_{U}$, and $\left(\operatorname{id}_{F U}-e_{U}\right) t_{U}^{n}\left(\operatorname{id}_{F U}-e_{U}\right) \rightarrow 0$ when $n \rightarrow \infty$ in $\operatorname{End}_{\mathscr{C}}(F U)$. Furthermore, $e$ is unique.

Now, define $F_{0}, F_{1}: \mathscr{U} \rightarrow \mathscr{C}$ by taking $F_{1} U$ and $F_{0} U$ to be summands of $F U$ corresponding to the idempotents $e_{U}$ and $\mathrm{id}_{F U}-e_{U}$, respectively (here we assume the axiom of choice for classes). (More precisely, for all $U \in \mathscr{U}$, choose summands $\left(S_{U}, i_{U}, p_{U}\right),\left(S_{U}^{\prime}, i_{U}^{\prime}, p_{U}^{\prime}\right)$ corresponding to $e_{U}, \mathrm{id}_{F U}-e_{U}$, respectively. For all $U, V \in \mathscr{U}$ and $f \in \operatorname{Hom}_{\mathscr{U}}(U, V)$, let $F_{1} U=S_{U}, F_{0} U=S_{U}^{\prime}$ and $F_{1} f=$ $p_{V} \circ F f \circ i_{U}, F_{0} f=p_{V}^{\prime} \circ F f \circ i_{U}^{\prime}$. Now, identify $F_{1} U \oplus F_{0} U=S_{U} \oplus S_{U}^{\prime}$ with $F U$ via $i_{U} \oplus i_{U}^{\prime}$.) Since $t_{U}$ commutes with $e_{U}$, it is a direct sum of two morphisms $\left(t_{0}\right)_{U} \in \operatorname{End}_{\mathscr{C}}\left(F_{0} U\right)$ and $\left(t_{1}\right)_{U} \in \operatorname{End}_{\mathscr{C}}\left(F_{1} U\right)$. It is easy to see that $F_{0}, F_{1}, t_{0}, t_{1}$ satisfy all requirements. That the decomposition $F=F_{0} \oplus F_{1}$ is unique up to natural isomorphism follows from the uniqueness of $e$.

Example 7.3. Let $\mathscr{C}$ be the category of finite abelian groups, which we make into a Hausdorff LT category by endowing it with the discrete topology $\left(\mathcal{I}_{\mathscr{C}}=\{0\}\right)$. Since the endomorphism ring of any object in $\mathscr{C}$ is finite, it has FP. Therefore, by Theorem 7.2 , for every functor $F: \mathscr{C} \rightarrow \mathscr{C}$ and a natural transformation $t$ : $F \rightarrow F$, we have an essentially unique decomposition $F=F_{0} \oplus F_{1}, t=t_{0} \oplus t_{1}$ where $t_{1}: F_{1} \rightarrow F_{1}$ is a natural isomorphism and $t_{0}: F_{0} \rightarrow F_{0}$ is a natural transformation which is nilpotent on every object. For example, in case $F$ is the identity functor and $t$ is multiplication by 2, the functors $F_{0}$ and $F_{1}$ are given by $F_{0} A=\{a \in A \mid \operatorname{ord}(a)$ is a power of 2$\}$ and $F_{1} A=\{a \in A \mid \operatorname{ord}(a)$ is odd $\}$ (where $\operatorname{ord}(a)$ denotes the order of $a$ in $A$ ).

\section{A Remark on Cancelation}

Let $\mathscr{C}$ be an additive category. An object $C \in \mathscr{C}$ is said to cancel from direct sums if $C \oplus C^{\prime} \cong C \oplus C^{\prime \prime}$ implies $C^{\prime} \cong C^{\prime \prime}$ for all $C^{\prime}, C^{\prime \prime} \in \mathscr{C}$. It is clear that all objects in a Krull-Schmidt category cancel from direct sums. In particular,

\footnotetext{
${ }^{9}$ If $\mathscr{C}$ is not small, the proof requires a stronger version of the axiom of choice, namely, that there exists a choice function for every class of nonempty classes. However, this can be avoided if there is a canonical way to choose the summand corresponding to an idempotent morphism in $\mathscr{C}$, which is indeed the case for many standard categories (e.g. module categories).
} 
this holds for the objects of $\operatorname{Rep}(Q, \mathscr{R})$ (with $Q$ a quiver and $\mathscr{R}$ a realization) if the assumptions of Theorem 6.2 apply. However, it turns out that these weaker condition holds under the much milder assumption that all objects in the categories $\left\{\mathscr{C}_{v}\right\}_{v \in Q_{0}}$ are semilocal ( $\mathscr{R}$ does not have to be linearly topologized).

The latter follows by applying two known results: The first is a theorem by Camps and Dicks (5) stating that a subring $S$ of a semilocal ring $R$ satisfying $S \cap R^{\times}=S^{\times}$(i.e. elements of $S$ that are invertible in $R$ are invertible in $S$ ) is semilocal. The second is the fact that modules with semilocal endomorphism ring cancel from direct sum. It is due to Evans and Bass: Evans ([6, Th. 2]) showed that if $M$ is an $R$-module and 1 is in the stable range of $\operatorname{End}_{R}(M)$, then $M$ cancels from direct sums; Bass ([3, Lm. 6.4]) showed that 1 is in the stable range of any semilocal ring. The Bass-Evans Theorem actually implies that in an additive category $\mathscr{C}$, every object $C$ whose endomorphism ring is semilocal cancels from direct sums. Indeed, assume $C \oplus C^{\prime} \cong C \oplus C^{\prime \prime}$ and let $E=C \oplus C^{\prime} \oplus C^{\prime \prime}$ and $R=\operatorname{End}_{\mathscr{C}}(E)$. It is well-know that the functor $\operatorname{Hom}_{\mathscr{C}}(E,-): \mathscr{C} \rightarrow \operatorname{Mod}-R$ is faithful and full once restricted to $\left.\mathscr{C}\right|_{E}$, the full subcategory of $\mathscr{C}$ consisting of summands of $E^{n}$ (with $n$ arbitrary). Since $C, C^{\prime},\left.C^{\prime \prime} \in \mathscr{C}\right|_{E}$, we may replace them with $\operatorname{Hom}(E, C), \operatorname{Hom}\left(E, C^{\prime}\right), \operatorname{Hom}\left(E, C^{\prime \prime}\right)$ and assume $\mathscr{C}=\operatorname{Mod}-R$. By Bass and Evans, we have $C^{\prime} \cong C^{\prime \prime}$ in this case.

Now let $\rho=\left(\left\{\mathscr{C}_{v}\right\},\left\{f_{a}\right\}\right) \in \operatorname{Rep}(Q, \mathscr{R})$ and assume $\operatorname{End}_{\mathscr{C}_{v}}\left(C_{v}\right)$ is semilocal for all $v \in Q_{0}$. Then $R:=\prod_{v} \operatorname{End}_{\mathscr{C}_{v}}\left(C_{v}\right)$ is semilocal. It is straightforward to check that every element of $\operatorname{End}(\rho)$ that is invertible in $R$ is invertible in $\operatorname{End}(\rho)$. Thus, by Camps and Dicks, End $(\rho)$ is semilocal. By Bass and Evans, this means $\rho$ cancels from direct sums, as required.

Note that, as in section 6, the result just established can be applied to obtain cancelation of representations of quivers on additive categories, twisted representations (in the sense of [9, §2]) and bilinear pairings. One should use the fact that the endomorphism ring of a finitely generated (resp. finitely presented) module over a commutative (resp. arbitrary) semilocal ring is semilocal by [7, Pr. 3.1] (resp. [7, Pr. 3.2]). The details are left to the reader.

\section{REFERENCES}

[1] Jiří Adámek, Horst Herrlich, and George E. Strecker. Abstract and concrete categories. Pure and Applied Mathematics (New York). John Wiley \& Sons Inc., New York, 1990. The joy of cats, A Wiley-Interscience Publication.

[2] Ibrahim Assem, Daniel Simson, and Andrzej Skowroński. Elements of the representation theory of associative algebras. Vol. 1, volume 65 of London Mathematical Society Student Texts. Cambridge University Press, Cambridge, 2006. Techniques of representation theory.

[3] H. Bass. K-theory and stable algebra. Inst. Hautes Études Sci. Publ. Math., (22):5-60, 1964.

[4] J.-E. Björk. Conditions which imply that subrings of semiprimary rings are semiprimary. $J$. Algebra, 19:384-395, 1971.

[5] Rosa Camps and Warren Dicks. On semilocal rings. Israel J. Math., 81(1-2):203-211, 1993.

[6] E. Graham Evans, Jr. Krull-Schmidt and cancellation over local rings. Pacific J. Math., 46:115-121, 1973.

[7] Alberto Facchini and Dolors Herbera. Local morphisms and modules with a semilocal endomorphism ring. Algebr. Represent. Theory, 9(4):403-422, 2006.

[8] Uriya A. First. Semi-invariant subrings. J. Algebra, 378:103-132, 2013.

[9] Peter B. Gothen and Alastair D. King. Homological algebra of twisted quiver bundles. $J$. London Math. Soc. (2), 71(1):85-99, 2005.

[10] Max Karoubi. K-theory. Springer-Verlag, Berlin, 1978. An introduction, Grundlehren der Mathematischen Wissenschaften, Band 226.

[11] Louis H. Rowen. Ring theory. Vol. I, volume 127 of Pure and Applied Mathematics. Academic Press Inc., Boston, MA, 1988.

[12] Seth Warner. Topological rings, volume 178 of North-Holland Mathematics Studies. NorthHolland Publishing Co., Amsterdam, 1993. 